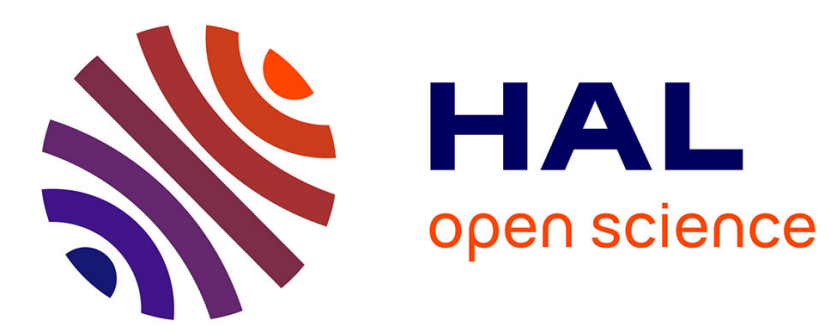

\title{
Fluctuation effects in the electrodynamic response of charge density wave condensates \\ L. Degiorgi
}

\section{To cite this version:}

L. Degiorgi. Fluctuation effects in the electrodynamic response of charge density wave condensates. Journal de Physique IV Proceedings, 1993, 03 (C2), pp.C2-103-C2-108. 10.1051/jp4:1993220 . jpa00251303

\section{HAL Id: jpa-00251303 https://hal.science/jpa-00251303}

Submitted on 1 Jan 1993

HAL is a multi-disciplinary open access archive for the deposit and dissemination of scientific research documents, whether they are published or not. The documents may come from teaching and research institutions in France or abroad, or from public or private research centers.
L'archive ouverte pluridisciplinaire HAL, est destinée au dépôt et à la diffusion de documents scientifiques de niveau recherche, publiés ou non, émanant des établissements d'enseignement et de recherche français ou étrangers, des laboratoires publics ou privés. 


\title{
Fluctuation effects in the electrodynamic response of charge density wave condensates
}

\section{DEGIORGI}

Laboratorium für Festkoerperphysik, ETH-Zürich, 8093 Zürich, Switzerland

\begin{abstract}
We report on the temperature dependence of the electrodynamic response of the CDW model compound $\mathrm{K}_{0.3} \mathrm{MoO}_{3}$ and its alloys. We found clear evidence of a pseudogap excitation and of the formation of a narrow Drude-like resonance at $T>T C D W$, as consequence of the precursor effects of the CDW phase transition. Moreover, we found signatures of the important role played by the thermal lattice fluctuations on the electrodynamic properties.
\end{abstract}

\section{Introduction}

The electrodynamics of the charge density wave ground state has been by now thoroughly explored, and several important features of this broken symmetry ground state have been identified [1]. The complete excitation spectrum of the CDW condensates is characterized by both the single particle gap $(\Delta)$ and the collective mode $\left(\omega_{0}\right)$ contribution to the frequency dependent conductivity. Furthermore, the study of the ac response in the $\mathrm{MHz}$ range is suggestive of different relaxation effects of the pinned mode due to the internal deformations of the CDW condensate [1]. It was also recognized that the optical response due to phonons is influenced by CDW formation through the electron-phonon coupling [1]. Recently, by combining various experimental techniques covering a broad spectral energy range, it was possible to identify a new resonance in the far infrared (FIR) above $\omega_{0}$ but below the typical phonon frequencies. This FIR mode was clearly recognized in the CDW compounds $\left(\mathrm{TaSe}_{4}\right)_{2} \mathrm{I}$ and $\mathrm{K}_{0.3} \mathrm{MoO}_{3}$, and, although weak, also in $\mathrm{TaS}_{3}[1,2,3]$.

Another central aspects of the phase transition, which occurs in low dimensional solids, is the appearance of fluctuation effects at temperatures above TCDW, where the three dimensional order develops, but below the mean field transition temperature $T_{M F}$ 
[4]. While fluctuation effects have been examined in various organic linear chain compounds, the question of whether collective transport effects (e.g. fluctuating or paraconductivity) associated with 1D fluctuations play an important role for $T_{C D W}<T<T_{M F}$ has remained an elusive problem. In this paper we address this problem and, particularly, we are interested in the deviation from a simple Drude behaviour slightly above TCDW, as consequence of the precursor effects of the CDW phase transition.

Another kind of fluctuations is related to the so called thermal lattice fluctuations. The zero-point lattice motion is of the same order of magnitude of the Peierls distortion and consequently one might expect possible huge effects on the electronic density of states [5]. Here, we shall consider the optical signatures or fingerprints, if any, of these thermal lattice fluctuations on the electrodynamic response.

\section{Experiment and Results}

The model CDW compound $\mathrm{K}_{0.3} \mathrm{MoO}_{3}$, so-called blue bronze, and its alloys $\mathrm{K}_{0.3} \mathrm{Mo}_{1-\mathrm{x}} \mathrm{W}_{\mathrm{x}} \mathrm{O}_{3}$ were used in our investigations. The blue bronze is an anisotropic metal with a chainlike crystal structure. The undoped material undergoes a metal-insulator transition at $T_{C D W}=180 \mathrm{~K}$, and structural studies clearly establish the development of an incommensurate CDW state, which occurs along the chain direction [1].

The optical reflectivity of large single crystals were measured in an extended photon energy range from $10^{5}$ down to $10 \mathrm{~cm}^{-1}$ using linearly polarized light (i.e. along the chain direction), in a temperature range between 5 and $300 \mathrm{~K}$. Four spectrometers were employed in order to cover such a large energy spectral range and particularly we made use of a Fourier spectrometer with a liquid He cooled Ge-bolometer in the FIR. The reflectivity evaluated over a broad energy spectral range has been used for the KramersKronig $(K K)$ transformation, from which we obtain the optical functions $(\varepsilon(\omega)$ and $\sigma(\omega))$. Details about the experimantal set up and data analysis are described in Ref. 1.

In order to give a taste of the obtained optical results, Fig. 1 presents the reflectivity spectra in the whole investigated frequency range at several significant temperatures (i.e., well below TCDW, around TCDW and at $300 \mathrm{~K}$ ), and the KK-obtained optical conductivity, respectively. In view of the issues we want to attack in the present discussion, we display in Fig. $2 \sigma_{1}(v)$ at several temperatures and in the frequency range, where the single particle CDW gap is expected (i.e., between 600 and 4900 $\mathrm{cm}^{-1}$ ), and in Fig. 3, on an expended scale, the relfectivity spectra and the corresponding $\sigma_{1}(v)$ in the FIR at temperatures around TCDW.

\section{Discussion}

In Fig. 1, we can monitor the phase transition and its influence in the optical properties. There is a clear change from a typical metallic optical signature (i.e. plasma edge behaviour in $R(v)$ and Drude-like one in $\left.\sigma_{1}(v)\right)$ to an excitation spectrum 


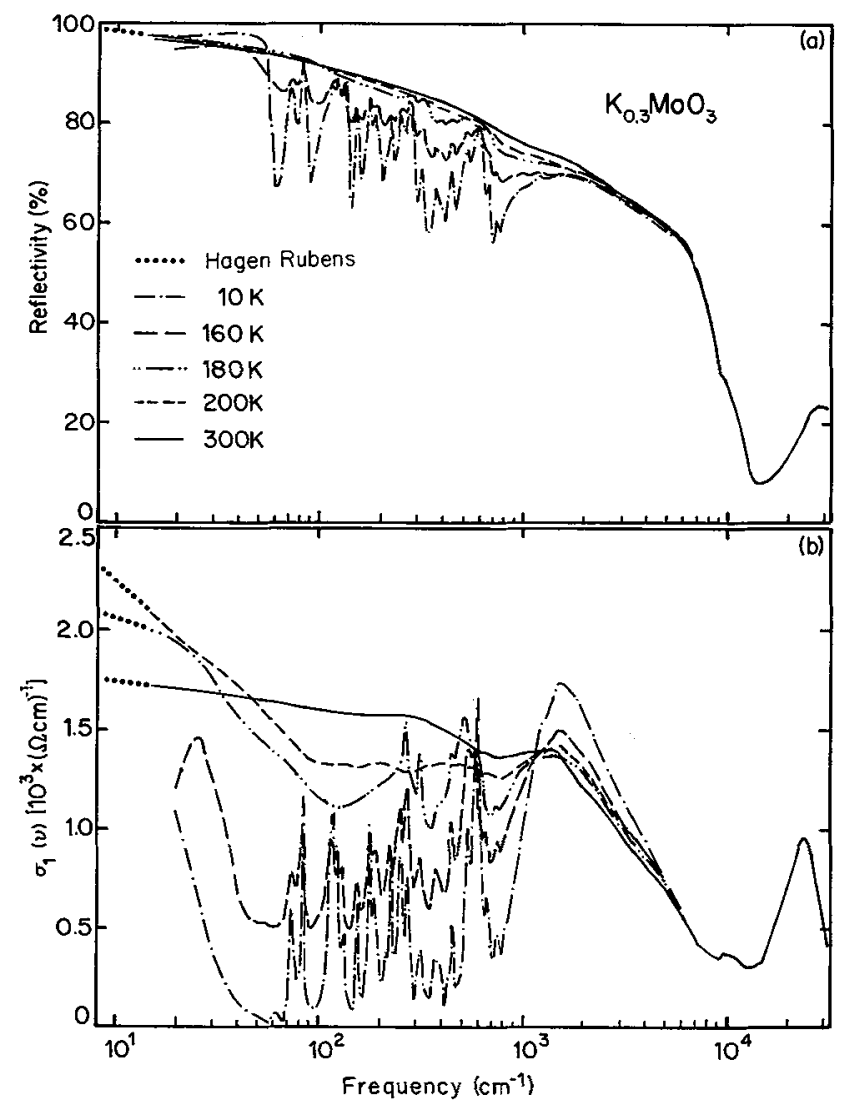

Fig.1: (a) Reflectivity spectra of $\mathrm{K}_{0.3} \mathrm{MoO}_{3}$ at several temperatures on the whole frequency range and (b) corresponding optical conductivity obtained through Kramers-Kronig transformations of the spectra in (a).

characteristic of an insulating phase. The various features characterizing the complete optical spectrum in the CDW ground state were already explained and discussed in Ref. 1 , and briefly sketched in the introduction.

The most remarkable features in Fig. 2 can be summarized as follows: (i) by lowering the temperature we observe a progressive shift of spectral weight from the low frequency range, which piles up at about $1500 \mathrm{~cm}^{-1}$, and (ii) the broadening of this aborption, which is ascribed to the single particle CDW gap, is larger than the energy scale $k_{B} T$. In fact, also at $10 \mathrm{~K}$ there is a substantial subgap tail. Recently, it has been shown that the zeropoint and the thermal lattice motions are a source of disorder: they have an effect on the electronic properties similar to that of a static random potential [5]. 


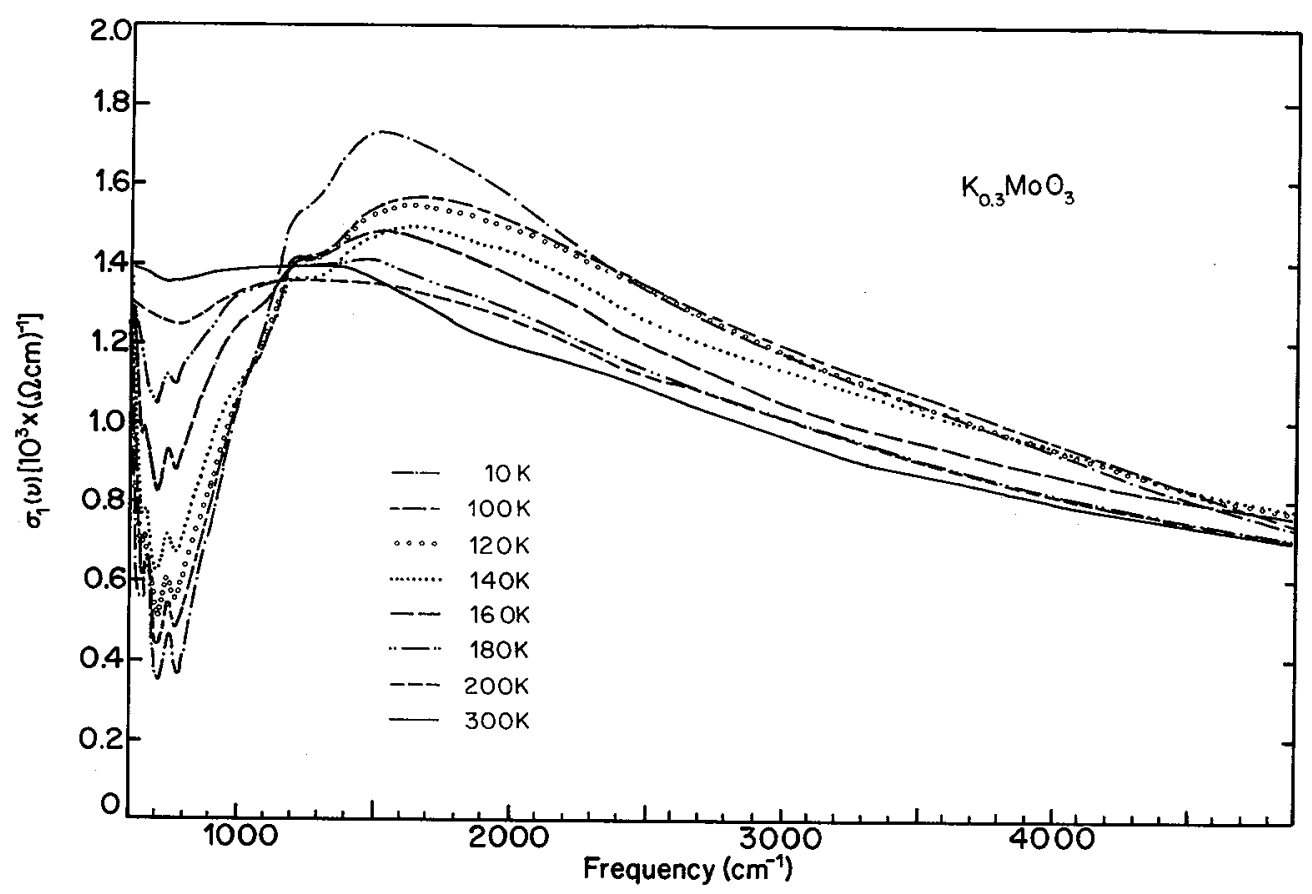

Fig.2: Optical conductivity at several temperatures in the frequency range between 600 and $4900 \mathrm{~cm}^{-1}$.

For this latter model exact expressions for the energy dependence of the electronic density of states can be obtained analytically. The thermal lattice fluctuations remove the inverse-square root singularity (as previously suggested by Lee, Rice and Anderson [6]) and produce a large tail of strongly localized states below the energy gap [5]. Preliminary calculations of the absorption spectrum are in fair agreement with our experimental results [7]. It is important to observe that the size of the electronic gap is proportional to the lattice distortion. Thus, in the case, where the zero point lattice motion is comparable to the Peierls distortion, one might wonder why there should be a clearly defined gap in the spectrum at all. Nevertheless, it can be shown that, when the band-width is much larger than the gap, as it usually is, the effect of the lattice fluctuations is reduced and a reasonably well-defined gap is still possible [5].

We now move to the discussion of the electrodynamic response of the CDW condensates around the CDW transition temperature. Fig. 3b shows the optical conductivity in the FIR at four temperatures, evaluated from the KK transformation of the $R(v)$ spectra [4]. We observe first of all that the dc limit of the optical conductivity agrees 
with the directly measured dc conductivity [1]. Second, the full spectral weight of the conductivity (integrated over the entire frequency range) is temperature independent within our accuracy of $\pm 2 \%$, as expected. The most interesting feature in Fig. $3 \mathrm{~b}$ is the formation of a narrow resonances at $\omega=0$, which develops at $200 \mathrm{~K}$. This resonance disappears below TCDW. We argue that this low frequency narrow resonance (at $200 \mathrm{~K}$ ) at the temperature where 1D fluctuations are important but 3D fluctuation effects do not play a significant role - corresponds to the one-dimensional unpinned CDW collective mode, and is a fingerprint of the fluctuation regime. Upon doping, this feature is progressively quenced. In fact, at moderate doping $(x<1 \%)$ the narrow resonance appears at lower temperatures, while at large doping $(x>2 \%)$ is completely suppressed. This is consistent with the dc-transport results [8], which show a progressive broadening of the phase transition upon doping.

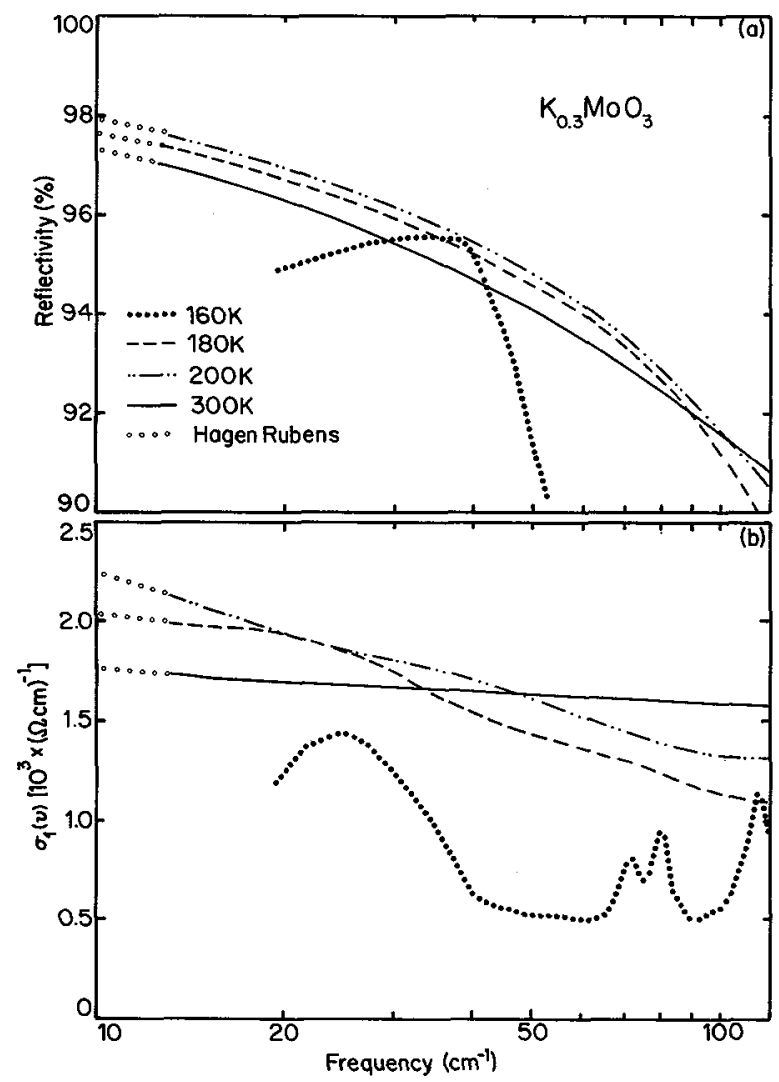

Fig.3: (a) Reflectivity spectra and (b) optical conductivity in the far-infrared at temperatures around TCDW. 
Another important manifestation of the fluctuating regime is also the gradual opening of the so called pseudogap and the temperature dependence of the magnetic susceptibility $\chi(T)$ has been accounted for by such a picture [9]. Indeed, the pseudo- (or effective) gap was found to be essentially temperature independent and below the CDW transition temperature the true gap at the same energy develops. We believe that the depression of $\sigma_{1}(v)$ (Fig. 1 and 2) around $700 \mathrm{~cm}^{-1}$ at $200 \mathrm{~K}$ (when compared with $\sigma_{1}$ at $300 \mathrm{~K}$ ) followed by a very broad hump at approximately $1000 \mathrm{~cm}^{-1}$ is due to the development of such a pseudogap. By lowering the temperature below TCDw, the hump at $1000 \mathrm{~cm}^{-1}$ is completely removed (Fig. 1 and 2), and a sharper and more intense structure gradually develops at the frequency of the CDW gap (i.e. at $1600 \mathrm{~cm}^{-1}[1]$ ).

\section{Conclusion}

Our thorough optical investigations of the electrodynamic response of the 1-dim. blue bronze and its alloys allow us to identify various features of the fluctuation regime (i.e. at temperature around or slightly above TCDW). The formation of a narrow Drude-like resonance and the appearence of a pseudogap (at $200 \mathrm{~K}$ ) are considered clear fingerprints of the fluctuation regime. Our results also confirm the important effect of zeropoint motion and thermal lattice fluctuations on the electrodynamic response of the CDW condensates. The substantial broadening of the CDW gap absorption is strongly indicative of the presence of localized states below the gap.

\section{Acknowledgements}

This work has been performed in collaboration with Prof. Grüner at UCLA. The author is also very grateful to Dr. R. McKenzie for valuable discussions, to Prof. Wachter at ETH for financial support, and to Mr. H.P. Staub and Mr. J. Müller for technical assistance.

\section{References}

[1] L. Degiorgi, B. Alavi, G. Mihaly and G. Grüner, Phys. Rev. B44, 7808 (1991)

[2] L. Degiorgi and G. Grüner, Europhys. Lett. 16, 97 (1991)

[3] L. Degiorgi and G. Grüner, Phys. Rev. B44, 7820 (1991)

[4] L. Degiorgi and G. Grüner, J. Phys. I (France) 2, 523 (1992)

[5] R.H. McKenzie and J.W. Wilkins, Phys. Rev. Lett. 69, 1085 (1992)

[6] P.A. Lee T.M. Rice and P.W. Anderson, Solid State Commun. 14, 703 (1974)

[7] R.H. McKenzie, private communication

[8] L.F. Schneemeyer, F.J. DiSalvo, S.E. Spengler and J.V. Waszczak, Phys. Rev. B30, 4297 (1984)

[9] D.C. Johnston, Phys. Rev. Lett. 52, 2049 (1984) 